\title{
The service design of GetSeverDirectory based on IEC 61850
}

\author{
Xianfan XU*, Qiang LI, Lei XU, Kun ZHANG \\ Key Lab of Intelligent Computing and Signal Processing of Ministry of Education, Anhui University \\ Hefei, China \\ e-mail:xxf@ahu.edu.cn
}

\begin{abstract}
MMS EASE Lite development package is always used to realize IEC 61850 protocol, but this conventional developing method has several drawbacks, such as high cost, huge data redundancy and no correspondence to IEC 61850 service. Therefore, a set of communication system which can meet IEC 61850 international standard is designed without using MMS EASE Lite. On that basis, SOCKET multithreaded method is used to realize GetSeverDirectory. The practical results showed that system prompted in this paper has the advantages of low cost, small data redundancy, fast transmission speed, credible data transmission, etc.
\end{abstract}

\section{Keywords-IEC61850;MMS;ASN.1;Service mapping}

\section{INTRODUCTION}

International standard IEC 61850, which is established by IEC (International Electrotechnical Commission), can be adapted to communication network and system of substation. The purpose is to realize interoperability and to share information between IEDs (Intelligent Electronic Device, IED can act as server or client) from different manufacturers by a standard protocol. The main characteristics are to model for equipments and objects and to conform power system communication service[1].

At present, when Chinese people develop our own intelligent electronic devices based on IEC 61850, introducing development package of MMS EASE Lite from Cisco Systems, Inc. will effectively shorten development cycle. But there are some problems. Firstly, the development package is very expensive ${ }^{2]}$. Secondly, the software package is huge because of the strong universality. So returning data redundancy is large when people use it. More processing power and more memory space are inevitably needed, which will undoubtedly increase development cost ${ }^{[3]}$. Again, due to the universality, many parts of the software package are not used when it is specific to a particular application ${ }^{\text {4] }}$. To some extent, this is a waste of source. People still need to carry out secondary development on that base since this software package is not specific to products of IEC $61850^{[5]}$. However, Chinese enterprises use development package of MMS EASE Lite, which is imported from abroad, to develop related products of IEC $61850^{[6][7]}$.

Based on above reasons, this paper researches MMS lower layer communication protocol and builds communication architecture applied to IEC 61850 communication service. Analyzing related protocols, coding and using SOCKET multi-thread method, GetSeverDirectory is realized. Other model services of IEC 61850 can be realized according to the method in this paper.

\section{SERVICE MODEL OF IED}

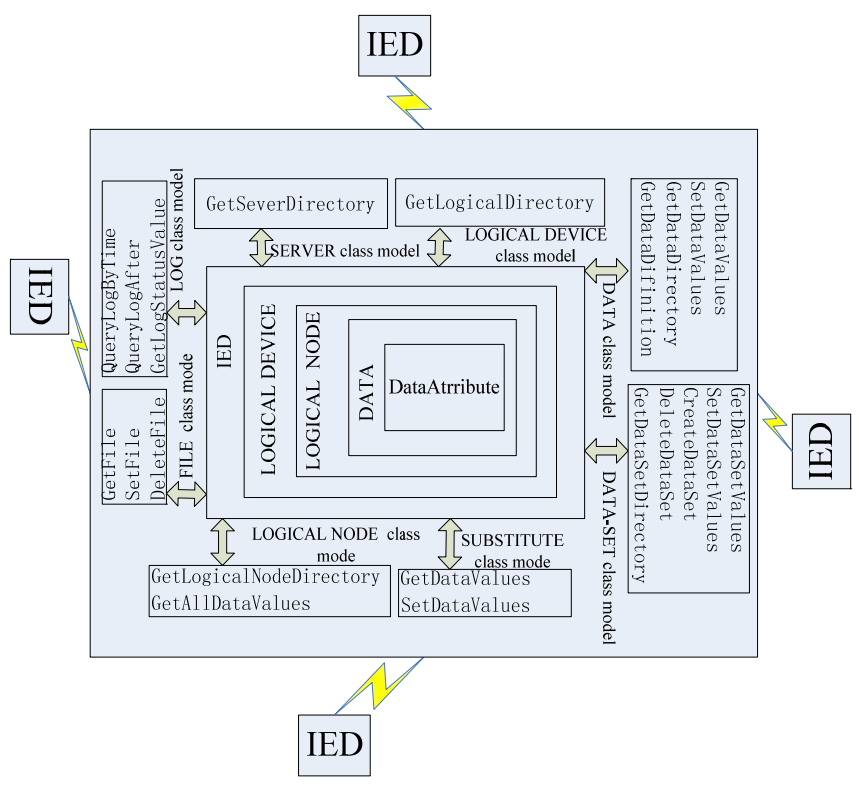

Figure 1. Service Model of IED

Interoperability and information sharing between IED are conducted by communication service. Internal structure of IED and related IEC 61850 communication services are showed in figure 1.There are multiple logical devices within IED and every logical device contains multiple logic nodes. The logical node can include mass data which has different attributes. IED Internal structure model used in this paper is built by analyzing IEC 61850 protocol. There are eight main model services, such as model service of server, SEVER class model, LOGICAL DEVICE class model, etc. Service of reading server directory can be realized by taking advantage of GetSeverDirectory in model service of the server.

\section{IEC 61850 COMMUNICATION FLOW}

IEC 61850 communication flow can be divided into there steps. The first step is using object-oriented approach to create IED internal structure and to analyze mapping from IEC 61850 service to MMS. It includes reading server directory, report, dataset and log, updating constant value, controlling selectively, etc. The second step is coding MMS sentence from application layer on presentation layer. 
According to IEC 8650, IEC 8825 and other protocols, realizing underlying communication by coding is also done. This part is the key of design because it involves implementing interoperability of device and accurately coding message which can ensure correct communication. A complete message consists of protocols in each layer and message. Coding results of related message are showed below. Coding involves a number of protocols which have different coding format and data package encapsulation format. So in this paper, message analysis and coding process are not described in detail because their complexity. The coding scheme is similar with reference [8]. The third step is transferring bit stream message with TCP on network.

\section{SYSTEM FRAMEWORK}

GetSeverDirectory is to know IED internal structure well. As is showed in figure 2, communication architecture of Ethernet+TCP/IP+RFC1006+MMS is created by analyzing related protocols, such as IEC $61850^{[9]}$, MMS $^{[10-14]}$, etc.

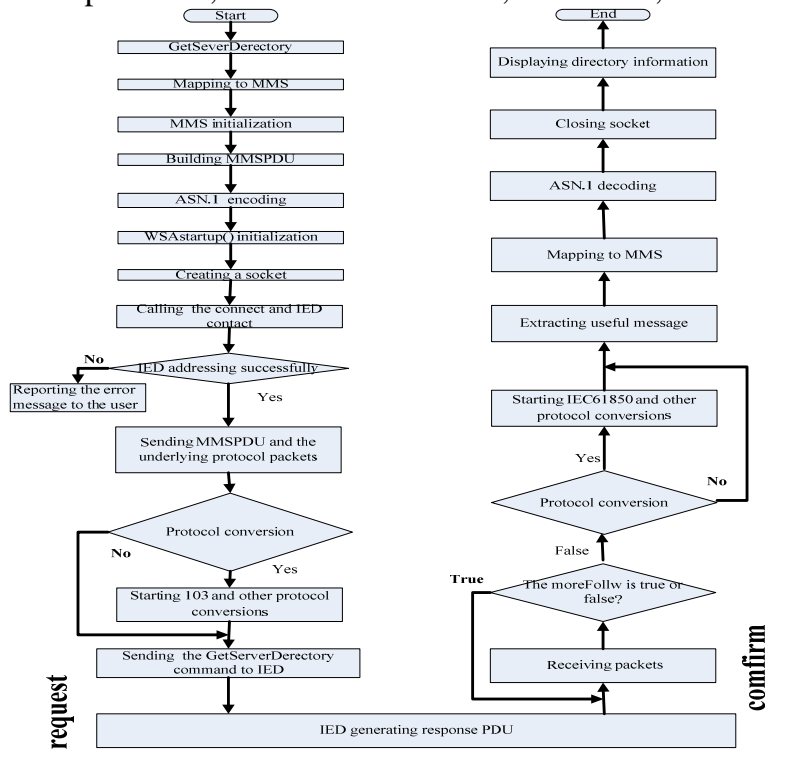

Figure 2. Communication Architecture

IEC61850 protocol realizes interaction between client and IED by using network to send/receive request, indication and response and to confirm service primitive. There are two communication service modes, service with confirmation and service without confirmation. The former is used in example of this paper.

A. Service with confirmation is showed in figure 3, including:

1) Client sends a request;

2) IED receives an indication;

3) IED performs related action;

4) If the actions are performed successfully, IED sends a positive answer

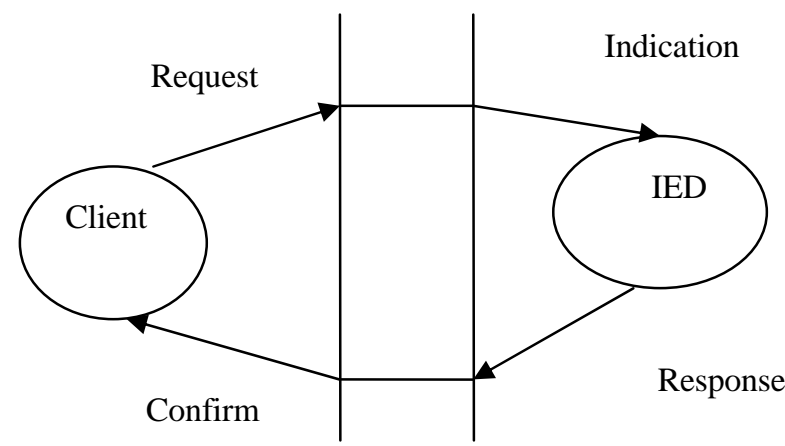

Figure 3. Service with Confirmation

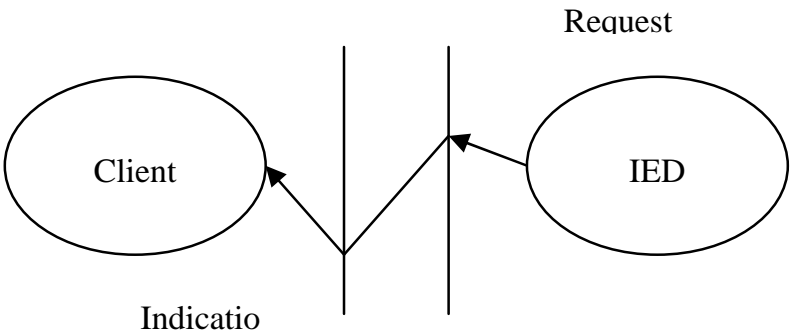

Figure 4. Service without Confirmation

B. As is showed in figure 4, service without confirmation sent by IED only consists of request and indication service primitive (information report, active state).

\section{SOFTWARE DESIGN}

GetSeverDirectory is mapped to MMS GetNamedList request. Data length of GetNamedList service is limited by the length of negotiable Manufacturing Message Specification Protocol Data Unit (MMSPDU). Data is segmented by return flag (moreFollow). If moreFollow value is TRUE, data is received continuously. Receiving does not end until the value is FLASE. Concrete flow chart is showed in figure 5 .

In order to assure accuracy of data transmission, this system uses socket stream, a connection-oriented data transmission method, which offers a bidirectional, reliable, orderly and repetitive way of transferring data. Data access between nodes on the network is realized by protocol data unit (PDU). This system uses ASN.1 encoding specification in PDU ${ }^{[15][16]}$. Using ASN.1 to describe PDU, ambiguity resulted in by normal language will be eliminated. Syntax format of ASN.1 is showed in table 1.

TABLE I. TABLE 1 SYNTAX FORM OF ASN.1

\begin{tabular}{|c|c|c|}
\hline Tag & Length & value \\
\hline
\end{tabular}




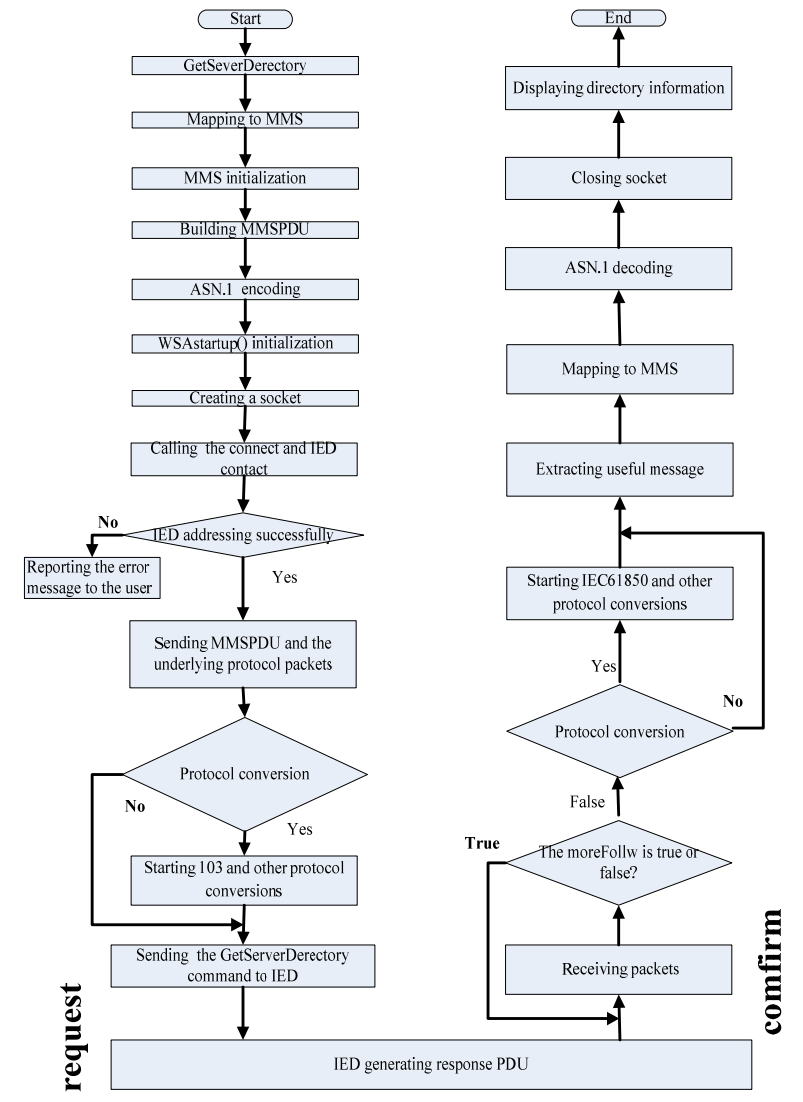

Figure 5. Flow Chart of GetSeverDirectory

"Tag" is tag type which represents the meaning of data. "Length" is data length. "Value" is data value. Communication with confirmation is mainly used in system.

1. Firstly, client will send a request of GetSeverDirectory, then according to this request and related parameters, MMSPDU will be built. Using ASN.1 to code MMSPDU, client will generate data stream which can transmit on the network. Finally, corresponding network transport protocols will reach IED equipments.

2. Secondly, bottom network transport service will use ASN.1 to decode received data stream and generate corresponding MMSPDU in IED. Service indication primitive with several parameters will be generated by decomposing MMSPDU. The parameters are equivalent to request primitive in semantics. IED will process the service primitive and return service response primitive with result and corresponding parameters. According to the returning service response primitive, data package conformed to syntax will be generated by ASN.1 coding. Finally, the data package will be returned to client by bottom network transport protocol.

3. Thirdly, back to client, the first thing is judging. If the last data of message in not 810100 (The value of moreFollow is FALSE), client will continue to receive message, else client will decode message, extract valid data and display it on the interface. Finally, GetSeverDirectory is finished.

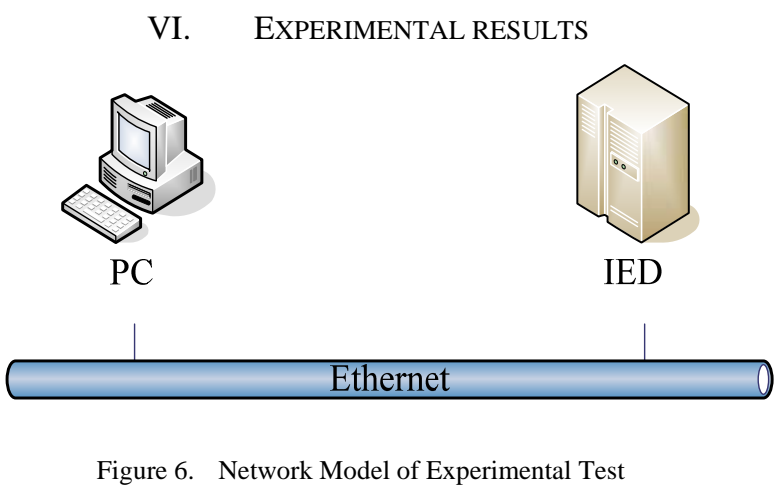

Network model of experimental test is showed in figure 6. According to network socket based on TCP/IP, communication with IED which have passed national IEC61850 conformance test under the VC environment. First, we build associated service (MMS initiate service must be called to build MMS environment before MMS communication session. ${ }^{[17]}$ ) to initialize MMS. Message built in MMS environment is showed as follows: a8 2680 0300 fd e8 8101 0a 82 01 0a 83010504168001018103 05 f1 0082 0c 03 ee 1c 00000408000079 ef 18. Reply message from IED is showed in figure 7.

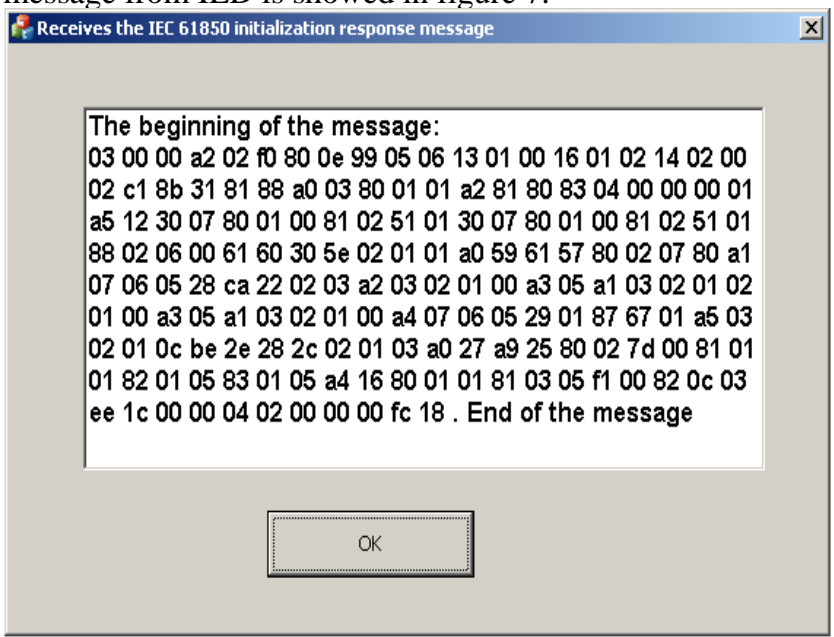

Figure 7. Building MMS Environment

As is showed in figure 7 , client receive initialization response message based on the framework in figure 2 . When IED receive MMS initialization request consisted of protocol RFC1006 、 8327 、8823 、 8650, it reply the message in figure 7 . In order to verify correctness of communication connection and accuracy of message coding, MMS Ethereal, which is the authoritative and professional analysis software (This software can capture real-time data packet and display service order in data packet, message corresponding to every protocol and decoding content of message, etc), is used to analyze message. The process is showed in figure 8. 


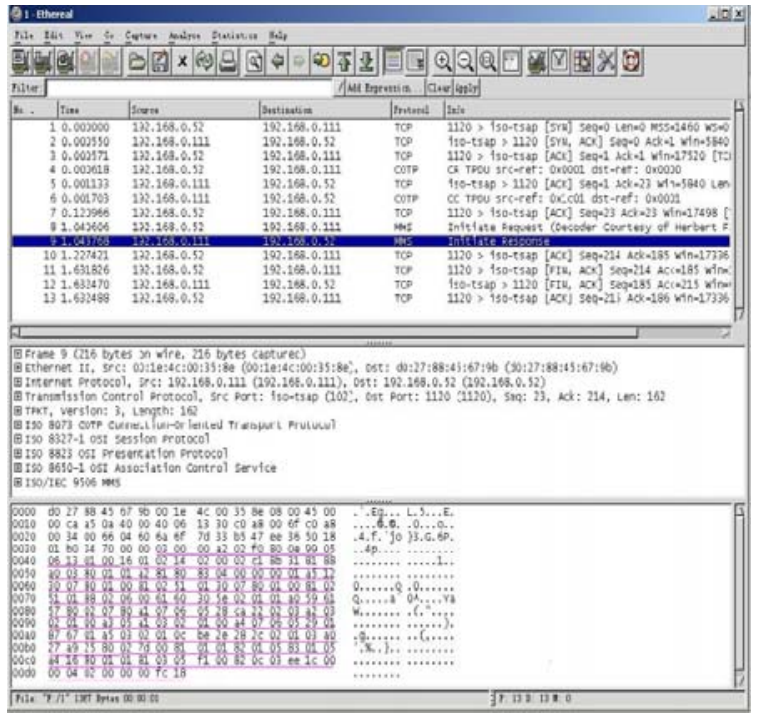

Figure 8. MMS Ethereal Software of Capturing Packet’s initialization

As is showed in figure 8, MMS Inititate Request in client has successfully initialized. Client also received MMS Inititate Response message which is same as figure 7. TCP/IP communication protocol specified that nine data packages should be exchanged between both of command operations at least. The former three packages finish TCP connection, then the latter two packages finish request and response, finally, the last four packages finish TCP closing. In order to transmit MMS protocol on TCP/IP, COTP data is sent. This figure displays the lifecycle of independent initialization on TCP/IP

If MMS initialized environment is built, any service of IEC 61850 can be operated. GetserverDirectory is mapped to MMS for Getnamelist Based on above built MMS environment, GetserverDirectory is conducted and GetnameList-Request message is sent. The request message is: a0 0e 020102 a1 09 a0 03800109 a1 0280 00. The practical results are showed in figure 9 .

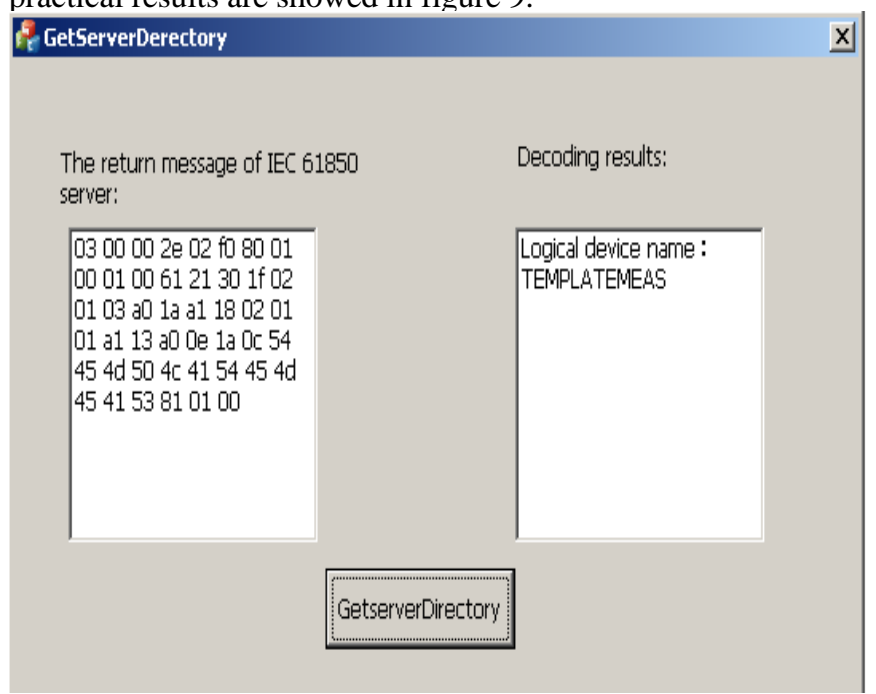

Figure 9. Realizing GetserverDirectory service
The GetnameList-Response message that is replied to client by IED is showed in left listbox of figure 9. The right listbox showed the ASN.1 decoding content of this message. In order to verify the correctness of message and the accuracy of decoding, MMS Ethereal software is used to capture data packet for analyzing. The process is showed in figure 10.

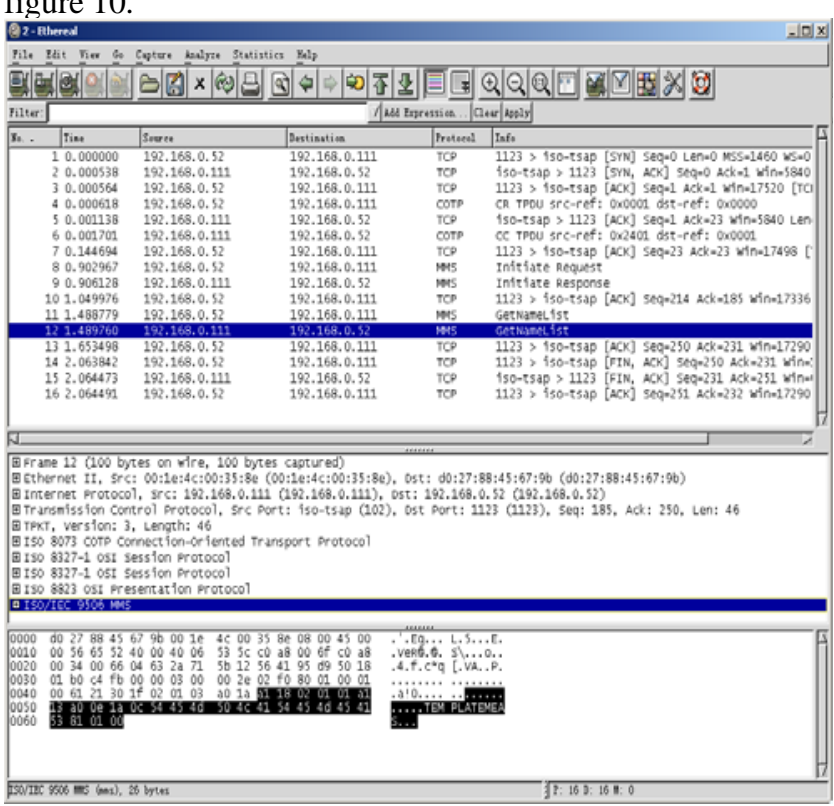

Figure 10. GetserverDirectory: the MMS Ethereal software of Capturing Packet

Figure 10 displays the lifecycle of GetserverDirectory. First, Connection between client and IED is realized by three handshakes of TCP; then MMS is initialized, GetserverDirectory is conducted after building initialized environment; finally, TCP connection is closed. The shaded part in the figure showed reply order of GetserverDirectory, message and decoding content which are same as figure 9. The results showed correctness of communication system framework in this paper, accuracy of data transmission and precision of protocol message's coding and decoding.

\section{CONCLUSION}

The paper attested the feasibility of developing IEC 61850 products independently without MMS EASE Lite development package. Comparing with using MMS EASE Lite development package, communication framework model prompted in the paper not only reduce development cost, increase accuracy of data transmission, reduce data redundancy, but also has advantages of simplifying communication process, reducing program code, speeding up every service response, optimizing the overall system.

\section{REFERENCE}

[1] Huang Wen-hua,Li Yong, "Research on Technology and Application of IEC 61850”, Modern Electronics Technique,2010.

[2] Zhang Ke-gang,Hou Si-zu,Chen Xiao-qian,Zhang Jie,"Research on the Gateway Transformation from IEC 60870-5-104 to IEC 
61850”,Telecommunications for Electric Power System,Vol.32 No.228,Oct.10,2011.

[3] Han Ming-feng,Zheng Yong-zhi,Tang Yong-jian, "Discussion and Implementation of IEC 61850 Directory and Definition Services”,Vol.30,No.18,Sept.25,2006.

[4] He Wei,Miao Wen-Gui,Zhu Song-yi, "Contadiction in the Mapping of IEC 61850 Mode and MMS and Its Solutions",Automation of Electric Power Systems,Vol.30,No.23,Dec.10,2006.

[5] Wang Li-hua,Wang Zhi-min,Ren Yan-ming,Su Yu Bin, "Design and Realization of IEC 61850 Communication Module Based on Plug-in Techique", Automation of Electric Power Systems,Vol.36,No.5,Mar.10,2012.

[6] Sun Jin-zhong, "Analying of implement the IEC 61850 model with MMS-EASE Lite”, Micro computer information,Vol.26,No.11,2010.

[7] Zhang Jie, Hou Si-zu, "Research on IEC 61850 Server Application Based on MMS-EASE Lite", Telecommunications for Electric Power System,Vol.32 No.227,Sep.10,2011.

[8] Wang De-wen,Zhang Chang-ming,Li Yuan,Dong Tao,"Study on data communication of a power telecontrol system based on ACSI/MMS gateway",Journal of North China Electric Power University,Vol.35,No.4,2008.

[9] IEC 61850-8-1,Communication Networks and Systems in Substations,Part 8-1:Specific Communication Service Mapping
(SCSM)-Mapping to MMS(ISO/IEC 9506 Part 1 and part 2) and to ISO/IEC 8802-3[S].

[10] ISO 9506,Industrial automation systems-Manufacturing message specification[S].ISO,2003.

[11] RFC1006, ISO transport services on top of the TCP:Version[S].ISO,1987.

[12] IEC 8073,Information processing systems-Open systems interconnection-Connection oriented transport protocol specification[S].ISO,1986.

[13] IEC 8327, Information processing systems-Open systems interconnection-Connection oriented Session protocol specification[S].ISO,1986.

[14] IEC 8823, Information processing systems-Open systems interconnection-Connection oriented Presentation protocol specification[S].ISO,1988.

[15] IEC 8824-1, Information Technology:Abstract Syntax Notation one [S].

[16] IEC 8825-1,Information Technology:ASN.1 Encoding Rules[S].

[17] IEC 8650, Information Technology -Open systems interconnectionConnection- oriented protocol for the Association Control Service Element-part1:Protocol specification[S].ISO,1996. 
Proceedings of the 2nd International Conference on Computer Science and Electronics Engineering (ICCSEE 2013)

Published by Atlantis Press, Paris, France.

(C) the authors

0346 
Proceedings of the 2nd International Conference on Computer Science and Electronics Engineering (ICCSEE 2013)

Published by Atlantis Press, Paris, France.

(C) the authors 0347 